\title{
Conserved Quantities for the Density Matrix and the Degree of Statistical Mixing
}

\author{
P. M. Radmore \\ Department of Electronic and Electrical Engineering, University College, Torrington Place. London, WC1E 7JE
}

Stephen M. Barnett,

Department of Engineering Science, University of Oxford, Parks Road, Oxford, OXI 3PJ

and

\section{A. M. Stoneham,}

Materials Physics and Metallurgy Division. Harwell Laboratory, UKAEA, Oxon, OX11 0RA

Received Octoher 3, 1989; accepted Octoher 8, 1989

\begin{abstract}
We investigate the conserved quantities for a closed, $N$-state quantum system. A simple derivation from the equation of motion for the density matrix shows that there is an infinite number of such conserved quantities. However, only the first $N$ of these constants of the motion are independent. These constants uniquely determine the degree of mixing in the system, which is itself conserved. We discuss the relationship between these results, the properties of Hamiltonians under unitary transformation and van Vleck's principle of spectroscopic stability.
\end{abstract}

\section{Introduction}

The density matrix $\rho$ for a closed $N$-state system obeys the Schrödinger equation

$i \hbar \frac{\partial \rho}{\partial t}=H \rho-\rho H=[H, \rho]$

where the Hamiltonian $H$ and $\rho$ are $N \times N$ matrices which may contain time-dependent elements. It has been shown that there exist $N$ conserved quantities associated with the Hamiltonian evolution of such a density matrix. These conservation laws are independent of the nature of the Hamiltonian and can be used to obtain general properties of $N$-level systems [1-5]. After giving a simple derivation of the $N$ conserved quantities, we show that they uniquely define the degree of statistical mixing in the initial state which is, in turn, a constant of the motion.

The conservation laws follow naturally from the unitary evolution of the density matrix. They arise from a general property of Hermitian matrices undergoing unitary transformations. We use this property to relate our results to van Vleck's principle of spectroscopic stability [6]. Further, the Hamiltonian under unitary transformation displays properties parallel to those of the density matrix under Hamiltonian evolution.

If the Hamiltonian contains a fluctuating indeterminate part then the conservation laws will, in general, only apply for individual realisations of the Hamiltonian. We discuss the failure of the conservation laws when the Hamiltonian fluctuations are statistically averaged.

\section{Conserved quantities}

We consider the density matrix $\rho$ for a closed $N$-state system obeying eq. (1). It is straightforward to show that any positive integral power of the density matrix, $\rho^{m}(m=1,2,3, \ldots)$, satisfies a similar equation:

$i \hbar \frac{\partial}{\partial t}\left(\rho^{m}\right)=\left[H, \rho^{m}\right]$.

To see that this is so, we write

$$
\begin{aligned}
\mathrm{i} \hbar \frac{\partial}{\partial t}\left(\rho^{m}\right) & =\mathrm{i} \hbar \sum_{n=0}^{m-1} \rho^{n} \frac{\partial \rho}{\partial t} \rho^{m-1-n} \\
& =\sum_{n=0}^{m-1} \rho^{n}(H \rho-\rho H) \rho^{m-1-n},
\end{aligned}
$$

using (1). The terms cancel in pairs leaving only $H \rho^{m}-\rho^{m} H$. The conserved quantities are found by taking the trace of the matrix eq. (2). Since the trace of the commutator of two finite matrices is zero, we find an infinite number of constants of the motion $C_{m}[1,2]$, where

$$
C_{m}=\operatorname{Tr}\left(\rho^{\prime \prime \prime}\right) \text {. }
$$

Clearly there cannot be an infinite number of independent conserved quantities since the system is finite. In fact, only the first $N$ constants are independent [4]. This can be readily shown by applying a unitary transformation to diagonalise the density matrix. We note that this is always possible because the density matrix is Hermitian but that the required transformation will be time-dependent. If we denote the diagonalised density matrix by $\tilde{\rho}$, with diagonal elements $\tilde{\rho}_{i}$ ( $i=1$ to $N$ ), then the conserved quantities (4) have the form

$$
\begin{gathered}
\tilde{\rho}_{1}+\tilde{\rho}_{2}+\cdots+\tilde{\rho}_{N}=C_{1} \\
\vdots \\
\vdots \\
\tilde{\rho}_{1}^{m}+\tilde{\rho}_{2}^{m}+\cdots+\tilde{\rho}_{N}^{m}=C_{m} \\
\vdots \\
\vdots \\
\tilde{\rho}_{1}^{N}+\tilde{\rho}_{2}^{N}+\cdots+\tilde{\rho}_{N}^{N}=C_{N} \\
\vdots \\
:
\end{gathered}
$$


The first constant $C_{1}$ equals unity by conservation of probability, but the other constants contain valuable statistical information. Equation (5) is an infinite set of simultaneous equations for the diagonalised density matrix elements $\tilde{\rho}_{i}$. The first $N-1$ equations may be used to eliminate $\tilde{\rho}_{1}, \rho_{2}, \ldots, \tilde{\rho}_{N-1}$. The $N$ th equation will then have the form of an $N$ th order polynomial for element $\tilde{\rho}_{N}$. The coefficients of this polynomial will be functions of only the first $N$ constants $C_{1}, C_{2}, \ldots, C_{N}$ and its $N$ roots will be the values of the diagonalised density matrix elements $\hat{\rho}_{i}$. Naturally, the order in which these solutions appear in $\tilde{\rho}$ is not prescribed. This order will be determined by the manner in which we label our eigenstates. Thus, the elements appearing in $\tilde{\rho}$ will be the same at all times but their arrangement may vary. It follows that only the first $N$ quantities are independent because the other constants $C_{n+1}, C_{N+2}, \ldots$ are expressible in terms of the $\tilde{\rho}_{i}$ which are themselves constants determined by the first $N$ conserved quantities.

\section{Conservation of mixing}

The density matrix allows us to treat systems in a mixed state for which we only know the probabilities that given pure states are occupied and for which the density matrix cannot be obtained from a wavefunction and its dual. The diagonal element $\tilde{\rho}_{i}$ is precisely the probability that the system is in the pure eigenstate of the instantaneous density matrix. These instantaneous basis states are complete and hence the $\tilde{\rho}_{i}$ contain all the information about the degree of statistical mixing in the system.

The $N$ diagonalised density matrix elements $\tilde{\rho}_{i}$ and the first $N$ conserved quantities are equivalent descriptions of the degree of statistical mixing. The constants $C_{1}, C_{2}, \ldots, C_{N}$ are related to the first $N$ moments of the distribution of the $\tilde{\rho}_{i}$, for

$$
C_{m}=\sum_{i=1}^{N} \tilde{\rho}_{i}^{m}=N\left\langle\tilde{\rho}_{i}^{m}\right\rangle,
$$

where $\left\langle\tilde{\rho}_{i}^{m}\right\rangle$ is the mean value of the quantities $\tilde{\rho}_{i}^{m \prime}(i=1$ to $N)$. In particular, the first conserved quantity gives, rather trivially, the mean of the $\tilde{\rho}_{i}$ :

$$
\left\langle\tilde{\rho}_{i}\right\rangle=\frac{1}{N} C_{1}=\frac{1}{N} .
$$

The second constant $C_{2}$ is related to the variance of the $\tilde{\rho}_{i}$ :

$$
\operatorname{var} \tilde{\rho}_{i}=\left\langle\tilde{\rho}_{i}^{2}\right\rangle-\left\langle\tilde{\rho}_{i}\right\rangle^{2}=\frac{C_{2}}{N}-\frac{1}{N^{2}} \text {. }
$$

The remaining constants $C_{3}, C_{4}, \ldots, C_{N}$ provide increasingly fine (and, ultimately, complete) statistical information. After the first $N$, the higher moments of the distribution of the $\tilde{\rho}_{i}$ are expressible in terms of $C_{1}, C_{2}, \ldots, C_{N}$.

The statistics of the mixing are uniquely determined by the first $N$ conserved quantities. Therefore the statistics or the degree of mixing of the system is conserved during the evolution. This result presents a generalisation of the wellknown statement that, in a Hamiltonian system, there is no mechanism by which a pure state can evolve into a mixed state (nor a mixed state into a pure state).

Physica Scripta 42

\section{Connection with the principle of spectroscopic stability}

The time-evolved density matrix $\rho(t)$ is related to the initial density matrix $\rho(0)$ by a unitary transformation:

$\rho(t)=U(t) \rho(0) U^{+}(t)$.

The conservation laws follow directly from (9):

$$
\begin{aligned}
\operatorname{Tr}\left(\rho^{\prime \prime}(t)\right) & =\operatorname{Tr}\left(U(t) \rho^{m}(0) U^{+}(t)\right) \\
& =\operatorname{Tr}\left(U^{+}(t) U(t) \rho^{\prime \prime}(0)\right) \\
& =\operatorname{Tr}\left(\rho^{\prime \prime \prime}(0)\right)
\end{aligned}
$$

where we have used the unitarity of $U(t)$ and the cyclic property of the trace operation. The conservation laws (10) depend only on the unitary form of the density matrix evolution. Therefore, similar laws will apply whenever an Hermitian matrix $M$ is transformed by the action of a unitary operator $S$ :

$\operatorname{Tr}\left(\left[S M S^{+}\right]^{m}\right)=\operatorname{Tr}\left(S M^{m} S^{+}\right)=\operatorname{Tr}\left(M^{m}\right)$.

Equation (11) contains van Vleck's principle of spectroscopic stability [6] which we now describe.

In its original form, the principle of spectroscopic stability was expressed as the invariance of a sum of matrix elements under changes of the system of quantisation. This sum of matrix elements occurs in the theory of susceptibilities. We present here a brief summary of van Vleck's analysis.

Consider an Hermitian operator $f^{0}$ with non-vanishing matrix elements $f^{0}\left(n^{\prime}, l^{\prime} ; n, l\right)$ between states with quantum numbers $n$ and $l$ and quantum numbers $n^{\prime}$ and $l^{\prime}$. The numbers $n$ (and $n^{\prime}$ ) describe sets of degenerate states characterised by the numbers $l$ (and $l^{\prime}$ ). The matrix elements of $f^{0}$ will determine transition rates for transitions induced by $f^{0}$. If a perturbation is applied to the system, or if we change from one system of quantisation to another, then the required transition rate will be determined by suitably modified matrix elements $f\left(n^{\prime}, l^{\prime} ; n, l\right)$. The perturbed operator $f$ will be related to the original operator $f^{0}$ by means of a unitary transformation,

$f=S^{\dagger} f^{0} S$,

which transforms $f$ from the original basis of degenerate quantum numbers $\left(l\right.$ and $l^{\prime}$ ) to a more suitable one. The principle of spectroscopic stability states that the sum over the degenerate quantum numbers of the transition rates from states $|n, l\rangle$ to $\left|n^{\prime}, l^{\prime}\right\rangle$ will be independent of the change in the basis of the degenerate states. Expressed mathematically, the principle states that

$\sum_{l . l^{\prime}}\left|f\left(n, l ; n^{\prime}, l^{\prime}\right)\right|^{2}=\sum_{l . l^{\prime}}\left|f^{0}\left(n, l ; n^{\prime}, l^{\prime}\right)\right|^{2}$.

In matrix notation, this equality becomes

$\operatorname{Tr}_{l}\left(f_{n, n} f_{n^{\prime}, n}^{*}\right)=\operatorname{Tr}_{l}\left(f_{n, n^{\prime}}^{0} f_{n^{\prime}, n}^{0 *}\right)$,

where $f_{n, t^{\prime}}$ is an operator promoting transitions from levels with quantum number $n^{\prime}$ to levels with quantum number $n$. The sum over degenerate quantum numbers $l^{\prime}$ in equation (13) becomes the matrix product in eq. (14), and the sum over $l$ becomes a trace. Expression (14) follows immediately from a trivial extension of eq. (11).

A simple example of the power of van Vleck's principle is the invariance of magnetic susceptibilities under the Paschenback effect [6]. The application of a magnetic field to an atom 
or molecule can lead to a re-organisation of Zeeman patterns but will not change the susceptibility: the position of any given Zeeman component of a spectrum involves only one value of the magnetic quantum number, whereas the calculation of a magnetic susceptibility requires a summation over all possible magnetic quantum numbers. This is analogous to the situation encountered with the statistical properties of the density matrix: the order in which the probabilities appear in the diagonalised density matrix is not prescribed but will depend on the manner in which the eigenstates are labelled. However, the statistical properties depend on the sums over all eigenstates and hence are unchanged by any relabelling.

Van Vleck's principle can be generalised to include all possible moments of $f$. This forms the basis of the method of moments [7, 8] which has been used in discussions of the effect of applied fields on optical absorption lineshapes for transitions between bands of degenerate or nearly degenerate levels.

The properties of Hermitian matrices under unitary transformation closely resemble those of the density matrix under Hamiltonian evolution. In particular, if the Hamiltonian is re-expressed in a different basis the eigenenergies of the Hamiltonian will be unaffected. This is the parallel statement to the invariance of the $\tilde{\rho}_{i}$ under unitary transformation. Further, just as the first $N$ moments of the density matrix (the traces of powers of $\rho$ ) determine all the statistical information about the system, so knowledge of the first $N$ moments of the Hamiltonian will determine the eigenenergies.

\section{Fluctuating Hamiltonians}

The density matrix evolution is constrained by the $N$ constants of the motion if the interaction is Hamiltonian, that is if $\rho$ obeys the Schrödinger equation. However, we must exercise caution if the interaction is fluctuating. In this case, any realisation of such an interaction will be Hamiltonian; however, averaging over many such realisations will not conserve the degree of statistical mixing. If the evolution of the ensemble is itself uncertain then the evolution of the density matrix will not be Hamiltonian:

$i \hbar \frac{\partial \bar{\rho}}{\partial t}=\overline{[\bar{H}, \rho]}$,

where the bar denotes averaging over the fluctuations of the Hamiltonian. This new density matrix $\bar{\rho}$ is still an accurate description of the statistical properties of the evolving system: the expectation value of an observable $A$ is

$$
\langle A\rangle=\overline{\operatorname{Tr}(\rho A)}=\operatorname{Tr}(\bar{\rho} A),
$$

provided $A$ is not itself fluctuating. The quantity $\operatorname{Tr}(\bar{\rho})$ is still conserved but the quantities $\operatorname{Tr}\left(\bar{\rho}^{m}\right)$, for $m>1$, may vary because of the non-Hamiltonian evolution of $\bar{\rho}$. For example

$$
\begin{aligned}
\mathrm{i} \hbar \frac{\partial}{\partial t}\left(\bar{\rho}^{2}\right) & =\mathrm{i} \hbar\left(\bar{\rho} \frac{\partial \bar{\rho}}{\partial t}+\frac{\partial \bar{\rho}}{\partial t} \bar{\rho}\right) \\
& =\bar{\rho} \overline{H \rho}-\overline{\rho H} \bar{\rho}+\overline{H \rho} \bar{\rho}-\bar{\rho} \overline{\rho H},
\end{aligned}
$$

using (15). The right-hand side of (17) is not, in general, expressible as a commutator. Therefore the trace of equation (17) need not vanish and consequently $\operatorname{Tr}\left(\bar{\rho}^{2}\right)$ will not be a constant. A similar conclusion will hold for the higher order traces.
As we have seen the quantities $C_{1}, \ldots, C_{N}$ are independent of the nature of the Hamiltonian and will be conserved even by a fluctuating interaction, for all realisations of the Hamiltonian, so that

$C_{i \prime \prime}=\operatorname{Tr}\left(\rho^{\prime \prime}\right)=\overline{\operatorname{Tr}\left(\rho^{\prime \prime \prime}\right)}$

However these are of limited use in this case because they are not simply related to the density matrix of interest $\bar{\rho}$.

We have noted that no Hamiltonian evolution could produce a mixed state from a pure state. However, averaging over a fluctuating Hamiltonian will induce uncertainty in the evolved density matrix. A simple example of this is the excitation of a single atom by a thermal light source. Our inability to provide more than a probabilistic description of the light results in a statistical uncertainty in the final state of the atom. The required averaging of the Hamiltonian destroys the Schrödinger evolution of the density matrix. A similar phenomenon occurs in the interaction between quantum systems when we discard information about one or more systems by tracing the complete density matrix over the unwanted degrees of freedom. The dynamics of the resulting reduced density matrix is non-Hamiltonian and may be described by a master equation [9].

Another example may be provided by the interaction of atoms with a phase-fluctuating laser [10]. There are then two situations in which we would need to carry out averaging. Firstly, if a single atom interacted with the laser field over a time which was long compared with the characteristic fluctuation time, then the result of a measurement on the atom would be averaged over the fluctuations. The density matrix for the atom would not then obey Hamiltonian evolution and the conservation laws would not hold. Secondly, if an experiment involved the interaction of many atoms (one at a time) with the field, each for a short time but with a total time again long compared with the fluctuation time, then a measurement obtained by averaging over the atoms would also be the result of non-Hamiltonian evolution. Of course, if the atom interacted with the field for a time short compared with the fluctuation time, then the conserved quantities would be preserved since the atoms, seeing an essentially fixed laser phase, would not be subject to a fluctuating Hamiltonian.

\section{Summary}

An $N$-state density matrix obeying a Hamiltonian equation of motion has $N$ independent constants of the motion, irrespective of the Hamiltonian. These conserved quantities correspond to the moments of the probability distribution for the system to be in a pure state. The statistics of the distribution are uniquely determined by these constants and the degree of statistical mixing of the system is therefore conserved during Hamiltonian evolution. The conservation laws follow directly from the unitary evolution of the density matrix. Indeed, similar laws hold for the unitary transformation of any Hermitian matrix. We have used this relationship to discuss the connection between our results and van Vleck's principle of spectroscopic stability. If we average a fluctuating interaction then the density matrix evolution will not be Hamiltonian and hence will not preserve the degree of mixing. 


\section{Acknowledgements}

Some of this work was carried out as part of the longer-term research within the Underlying Programme of the UKAEA. S. M. B. thanks G. E. C. and the Fellowship of Engineering for the award of a Senior Research fellowship.

\section{References}

1. Elgin, J. N., Phys. Rev. Lett. 80A, 140 (1980).

2. Hioe, F. T. and Eberly, J. H., Phys. Rev. Lett. 47, 838 (1981); Phys. Rev. A25, 2168 (1982)

3. Gottlieb, H. P. W., Phys. Rev. A26, 3713 (1982); Phys. Rev. A32, 653 (1985).
4. Hioe, F. T., Phys. Rev. A29, 3434 (1984).

5. Aravind, P. K., J. Opt. Soc. Am. B3, 1025 (1986).

6. Van Vleck J. H.. "The Theory of Electric and Magnetic Susceptibilities", Oxford University Press (1932).

7. Henry, C. H., Schnatterly, S. E. and Slichter, C. P., Phys. Rev. 137, $583(1965)$.

8. Stoneham, A. M., "Theory of Defects in Solids", Oxford University Press (1975).

9. Louisell, W. H., "Quantum Statistical Properties of Radiation", Wiley (1973).

10. Sargent, M., Scully, M. O. and Lamb, W. E., "Laser Physics", Addison-Wesley (1974). 